\title{
Convex approximations for a class of mixed-integer recourse models
}

\author{
Maarten H. Van der Vlerk
}

Published online: 18 July 2009

(C) The Author(s) 2009. This article is published with open access at Springerlink.com

\begin{abstract}
We consider mixed-integer recourse (MIR) models with a single recourse constraint. We relate the second-stage value function of such problems to the expected simple integer recourse (SIR) shortage function. This allows to construct convex approximations for MIR problems by the same approach used for SIR models.
\end{abstract}

Keywords Mixed-integer recourse · Convex approximation

\section{Introduction}

Consider the mixed-integer recourse model

$$
\begin{array}{ll}
\min _{x} & c x+\mathcal{Q}(x) \\
\text { s.t. } & x \in X:=\left\{x \in \mathbb{R}_{+}^{n-n^{\prime}} \times \mathbb{Z}_{+}^{n^{\prime}}: A x \geq b\right\}
\end{array}
$$

where

$$
\mathcal{Q}(x):=\mathbb{E}_{\omega}[v(\omega-T x)], \quad x \in \mathbb{R}^{n},
$$

and, for $s \in \mathbb{R}$,

$$
\begin{array}{ll}
v(s):=\min _{y, z} q y+\bar{q} z \\
\text { s.t. } \quad w y+\bar{w} z \geq s \\
& (y, z) \in C \\
& y \in \mathbb{R}_{+}^{m-m^{\prime}}, \quad z \in \mathbb{Z}_{+}^{m^{\prime}}
\end{array}
$$

M.H. Van der Vlerk $(\bowtie)$

Department of Operations, University of Groningen, P.O. Box 800, 9700 AV Groningen, The Netherlands

e-mail: m.h.van.der.vlerk@rug.nl 
The function $v$ is the second-stage value function, and the function $\mathcal{Q}$ is called the expected value function. These functions model the (expected) costs of recourse actions to compensate for infeasibilities associated with the random goal constraint $T x \geq \omega$. The right-hand side parameter $\omega$ is a random variable with known cumulative distribution function (cdf) $F_{\omega}$.

This model has only a single recourse constraint, i.e., $w$ and $\bar{w}$ are vectors, and $s \in \mathbb{R}$. In addition, there may be further linear constraints on the second-stage variables $y$ and $z$, but they do not involve the first-stage decisions $x$ nor the random parameter $\omega$. Such constraints are denoted by $(y, z) \in C$.

Our main motivation to study this model is that it is the simplest extension of pureinteger recourse models, which we studied in a number of papers (Klein Haneveld et al. 1996, 2006; Louveaux and Van der Vlerk 1993; Van der Vlerk 2004). In particular, we will see that the approach which we developed to construct convex approximations for the recourse function $\mathcal{Q}$ in the pure-integer case, can be extended to this mixed-integer recourse model. For a general description of this modification of recourse data approach, see Van der Vlerk (2003).

In addition, this recourse model (with $q_{i}>\bar{q}_{j}, i=1, \ldots, m-m^{\prime}, j=1, \ldots, m^{\prime}$ ) can be interpreted as a production planning problem. Using inputs $x$ and given technological constraints $x \in X$, we wish to produce some good $T x$ to meet uncertain future demand $\omega$, so as to minimize total expected costs $c x+\mathcal{Q}(x)$. In case production falls short of demand, recourse actions $y$ and $z$ can be used to compensate the shortage. The integer variables $z$ represent batches of various sizes $\bar{w}$ (e.g., amounts bought from competitors), whereas the continuous variables $y$ denote 'fractional' but more expensive production. We will return to this interpretation at the end of Sect. 2.

For a general introduction to recourse models we refer to the textbooks (Birge and Louveaux 1997; Kall and Wallace 1994; Prékopa 1995), the handbook (Ruszczynski and Shapiro 2003), and the website. ${ }^{1}$ Structural properties of mixed-integer recourse models were studied in Schultz (1993, 1995), and first algorithms are described in Carøe and Tind (1997), Laporte and Louveaux (1993), Norkin et al. (1998). Surveys of properties, algorithms, and applications for (mixed-)integer recourse models can be found in Klein Haneveld and Van der Vlerk (1999), Louveaux and Schultz (2003), Stougie and Van der Vlerk (1997, 2003). Stochastic (mixed-)integer programming has received increasing research attention during the last years. For example, (Dyer and Stougie 2006; Swamy and Shmoys 2005) are contributions from a computer science perspective. In Alonso-Ayuso et al. (2003), multi-stage problems are solved by branch-and-fix, using the non-anticipativity constraints to coordinate the procedure. Decomposition, and various techniques to transform cuts obtained for a single subproblem to valid inequalities for all subproblems, underly the algorithms proposed in Guan et al. (2005), Sen and Higle (2005), Sen and Sherali (2006), Sherali and Zhu (2006). In Kong et al. (2006) an equivalent superadditive dual formulation is exploited to solve two-stage pure-integer problems. A branch-andprice algorithm is proposed in Silva and Wood (2006) to solve a stochastic facility-location problem. Finally, (Schultz and Tiedemann 2003; Schultz and Tiedemann 2006) consider mean-risk versions of mixed-integer recourse problems.

The remainder of this paper is organized as follows. In Sect. 2 we analyze the secondstage value function $v$, and show that it can be represented as an expected integer shortage function. The latter function was studied extensively in the context of simple integer recourse models. In Sect. 3 we use this knowledge to come up with convex approximations of the

\footnotetext{
${ }^{1}$ Stochastic Programming Community Home Page sponsored by COSP. http://stoprog.org.
} 
recourse function $\mathcal{Q}$, and show that such approximations can be represented by continuous simple recourse functions. Section 4 contains a discussion on solution approaches, and a summary and concluding remarks follow in Sect. 5.

\section{Analysis of the second-stage value function}

In addition to the standard assumption that the second-stage value function $v$ is finite-valued (i.e., the recourse is complete and sufficiently expensive), we assume

(i) $v(t)=0$ for $t \leq 0$ and $v(t)>0$ for $t>0$.

(ii) For some period $p>0$, the function $v$ satisfies

$$
v(k \cdot p+t)=k \cdot v(p)+v(t), \quad 0 \leq t<p, k \in \mathbb{Z}_{+} .
$$

We say that $v$ is semi-periodic on $\mathbb{R}_{+}$: on this interval $v$ is the sum of a periodic function with period $p$ and a linear function with slope $v(p)$.

Clearly, assumption (ii) means a further restriction of the class of models under study. However, it is satisfied by e.g. simple recourse models (see Fig. 1), and more generally, if there exists a largest batch of size $p$ which provides the cheapest way of compensating a shortage $p$.

To simplify the presentation, we assume that the period $p=1$ and that $v(p)=1$, which always can be obtained by scaling. Then

$$
v(s)= \begin{cases}0, & s \leq 0, \\ \lfloor s\rfloor+v(\langle s\rangle), & s \geq 0,\end{cases}
$$

where $\langle s\rangle:=s-\lfloor s\rfloor$ is the fractional part of $s \in \mathbb{R}$.

Observing that $v(0)=0, v(1)=1$, and that $v$ is non-decreasing on $[0,1]$ and lower semi-continuous (hence left-continuous), we associate $v$ with the left-continuous cumulative distribution function (cdf) $F_{v}(s):=\operatorname{Pr}\{v<s\}$ of a random variable $v$ with support in [0,1],

$$
F_{v}(s):= \begin{cases}0, & s \leq 0, \\ v(s), & s \in[0,1], \\ 1, & s \geq 1 .\end{cases}
$$

Then $v(s)=\lfloor s\rfloor^{+}+F_{v}(\langle s\rangle), s \in \mathbb{R}$, with $\lfloor s\rfloor^{+}:=(\lfloor s\rfloor)^{+}$denoting the positive part of $\lfloor s\rfloor$. Analogously, we define $\lfloor s\rfloor^{-}:=(\lfloor s\rfloor)^{-}$as the negative part of $\lfloor s\rfloor$, and $\lceil s\rceil^{+}:=(\lceil s\rceil)^{+}$.
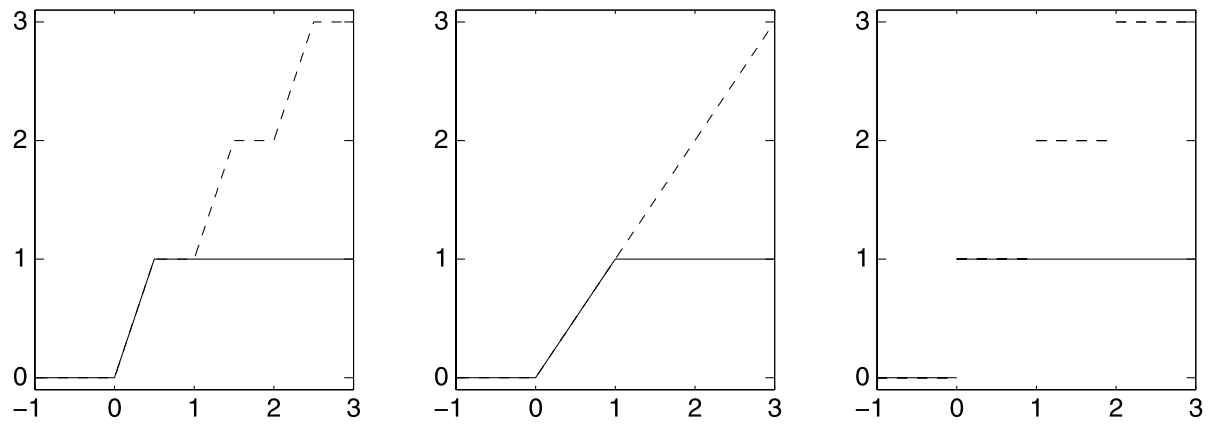

Fig. 1 The MISR function $v$ (dashed) and corresponding cdf $F_{v}$ of Example 1 , for $q \in\{2,1, \infty\}$ 
Example 1 Define the (one-dimensional) mixed-integer simple recourse (MISR) value function as

$$
v(s)=\min \left\{q y+z: y+z \geq s, y \in \mathbb{R}_{+}, z \in \mathbb{Z}_{+}\right\}, \quad s \in \mathbb{R},
$$

with $q \geq 1$. Then $v(s)=\min \left\{\lfloor s\rfloor^{+}+q\langle s\rangle,\lceil s\rceil^{+}\right\}$, so that $F_{v}$ is the cdf of the continuous uniform distribution on $[0,1 / q]$ (notation: $v \sim U(0,1 / q)$ ).

Continuous simple recourse corresponds to MISR with $q=1$, and has $v(s)=(s)^{+}$, $s \in \mathbb{R}$, so that $F_{v}$ is the cdf of $v \sim U(0,1)$.

With $q=+\infty$ we obtain pure integer simple recourse, with $v(s)=\lceil s\rceil^{+}, s \in \mathbb{R}$, so that $F_{v}$ is the cdf of the degenerated random variable $v$ with $\operatorname{Pr}\{v=0\}=1$.

Figure 1 shows these MISR functions $v$ and corresponding $F_{v}$.

Lemma 1 Consider a value function $v$ satisfying (1), and let $v$ be a random variable with associated cdf $F_{v}$ according to (2). Then

$$
v(s)=\mathbb{E}_{v}\left[\lfloor v-s\rfloor^{-}\right], \quad s \in \mathbb{R} .
$$

Proof For $s \leq 0$ the result follows trivially.

The random variable $v$ takes values in $(0,1]$. Hence, for any fixed $s \in \mathbb{R}$, the random variable $s-v$ takes values in the interval $[s-1, s)$, which contains precisely one integer value $\lfloor s\rfloor$. Thus, $\lceil s-v\rceil$ is a two-valued random variable,

$$
\lceil s-v\rceil= \begin{cases}\lfloor s\rfloor, & \text { if } s-v \leq\lfloor s\rfloor \\ \lfloor s\rfloor+1, & \text { if } s-v>\lfloor s\rfloor\end{cases}
$$

so that

$$
\mathbb{E}_{v}[\lceil s-v\rceil]=\lfloor s\rfloor+1 \cdot \operatorname{Pr}\{v<\langle s\rangle\}=\lfloor s\rfloor+F_{v}(\langle s\rangle),
$$

which is equal to $v(s)$ for $s>0$. For such $s$, observing that $s-v>-1$ so that $\lceil s-v\rceil=$ $\lceil s-v\rceil^{+}$, the result follows since $\lceil t\rceil^{+}=\lfloor-t\rfloor^{-}, t \in \mathbb{R}$.

We conclude that each value function $v$ under consideration is equivalent to an integer expected shortage function

$$
H(s):=\mathbb{E}_{v}\left[\lfloor v-s\rfloor^{-}\right], \quad s \in \mathbb{R},
$$

where the expectation is taken with respect to a random variable $v$ whose distribution captures the specific properties of $v$. The integer expected shortage function is well-studied in the context of simple integer recourse models. In the next section we will use its properties to derive convex approximations for the expected recourse function $\mathcal{Q}$.

As mentioned in the Introduction, our recourse model can be seen as a production planning model. In that context, Lemma 1 has an interesting interpretation. On the one hand, $v(s)$ represents the minimal costs for satisfying deterministic demand $s$, using a relatively sophisticated technology with various batch sizes as well as 'fractional' production, all with corresponding unit costs. By Lemma 1, the same expected production costs can be obtained by using a very simple production technology, which allows only a single unit batch size. Indeed, if one introduces a suitable random disturbance $v$, and aims to satisfy the stochastic 
demand $s-v$, then on average the production costs will be the same for these two models. From the producer's perspective, this means that savings on the costs for installing production technology can be obtained. On the other hand, clients may not be happy with the outcomes of such a 'virtual' production technology.

\section{The recourse function}

We now turn to studying the mixed-integer recourse function $\mathcal{Q}$, which is defined as $\mathcal{Q}(x)=$ $\mathbb{E}_{\omega}[v(\omega-T x)]$ for $x \in \mathbb{R}^{n}$, where the random variable $\omega$ represents stochastic demand. To derive properties of this function, we can equivalently study it as a function of the tender variable $T x \in \mathbb{R}$. To this end, we define the one-dimensional function

$$
Q(x):=\mathbb{E}_{\omega}[v(\omega-x)], \quad x \in \mathbb{R} .
$$

\subsection{Properties}

By Lemma 1, we have, for $x \in \mathbb{R}$,

$$
\begin{aligned}
Q(x) & =\mathbb{E}_{\omega}\left[\mathbb{E}_{v}\left[\lfloor v-(\omega-x)\rfloor^{-}\right]\right] \\
& =\mathbb{E}_{v}\left[\mathbb{E}_{\omega}\left[\lfloor-\omega+(x+v)\rfloor^{-}\right]\right] \\
& =\mathbb{E}_{v}\left[\mathbb{E}_{\omega}\left[\lceil\omega-(x+v)\rceil^{+}\right]\right] \\
& =\mathbb{E}_{v}[G(x+v)],
\end{aligned}
$$

where $G(t):=\mathbb{E}_{\omega}\left[\lceil\omega-t\rceil^{+}\right], t \in \mathbb{R}$, is the integer expected surplus function. This function $G$ is the counterpart of the integer expected shortage function discussed above, and was also studied in the context of simple integer recourse models.

Alternatively, defining the random variable $\delta:=\omega-v$, we obtain

$$
Q(x)=\mathbb{E}_{\delta}\left[\lceil\delta-x\rceil^{+}\right], \quad x \in \mathbb{R},
$$

where $\delta$ has cdf $F_{\delta}$,

$$
F_{\delta}(t)=\int_{0}^{1} F_{\omega}(t+s) d F_{v}(s), \quad t \in \mathbb{R}
$$

The identities (3) and (4) show that properties of the mixed-integer recourse function $Q$ follow trivially from those of the integer expected surplus function $G$. Moreover, they provide an easy way to evaluate $Q$, given the formula

$$
G(t)=\sum_{k=0}^{\infty}\left(1-F_{\omega}(t+k)\right), \quad t \in \mathbb{R} .
$$

For the derivation of this formula and properties of the function $G$, we refer to Louveaux and Van der Vlerk (1993) and Van der Vlerk (1995). 
Fig. 2 The function $Q$ of Example 2

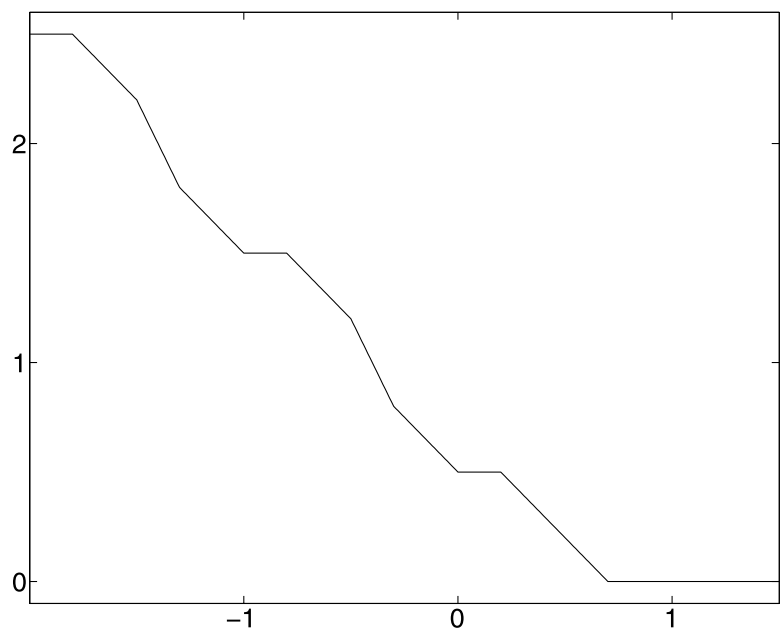

Example 2 Consider the MISR value function $v(s)=\min \left\{\lfloor s\rfloor^{+}+q\langle s\rangle,\lceil s\rceil^{+}\right\}, s \in \mathbb{R}$. As shown in Example 1, for finite $q \geq 1$ the associated cdf $F_{v}$ is that of the uniform distribution on $[0,1 / q]$, so that

$$
F_{\delta}(t)=q \int_{0}^{1 / q} F_{\omega}(t+s) d s, \quad t \in \mathbb{R} .
$$

It follows that the random variable $\delta$ has a probability density function (pdf) $f_{\delta}$,

$$
f_{\delta}(t)=q\left(F_{\omega}(t+1 / q)-F_{\omega}(t)\right), \quad t \in \mathbb{R} .
$$

For example, assuming $q=2$ and that $\omega$ is discrete with equally likely realizations 0 and 0.7 , it follows by straightforward computation that $\delta$ is uniformly distributed on two disjunct intervals:

$$
f_{\delta}(t)= \begin{cases}1, & t \in[-0.5,0] \cup[0.2,0.7] \\ 0, & \text { otherwise }\end{cases}
$$

See Fig. 2 for the graph of the MISR recourse function $Q(x)=\mathbb{E}_{\delta}\left[\lceil\delta-x\rceil^{+}\right], x \in \mathbb{R}$.

We are particularly interested in convexity of the recourse function $Q$. From (3) and (4) it is clear, that this is directly related to convexity of the function $G$. In Klein Haneveld et al. (2006) it is shown that $G$ is convex if and only if the underlying random variable $\omega$ has a pdf $f$ satisfying

$$
f(t)=F(t+1)-F(t), \quad t \in \mathbb{R},
$$

where $F$ is an arbitrary cdf with finite mean value.

Corollary 1 Consider a value function $v$ satisfying (1), and let $v$ be a random variable with associated cdf $F_{v}$ according to (2). Then the recourse function $Q(x)=\mathbb{E}_{\omega}[v(\omega-x)]$, $x \in \mathbb{R}$, is convex if and only if the random variable $\delta:=\omega-v$ has a pdf satisfying (6).

In particular, $Q$ is convex if $\omega$ has a pdf satisfying (6). 
Remark 1 It is well-known that the continuous simple recourse function $Q$, i.e., the special case of MISR with $q=1$, is convex for every distribution of $\omega$. This indeed follows trivially from Corollary 1 and (5).

Corollary 1 shows that the mixed-integer recourse function $Q$ is convex only in exceptional cases. Therefore, we are interested in convex approximations of this function, which is the subject of the next section.

\subsection{Convex approximations}

Corollary 1 suggest that, to obtain convex approximations of a non-convex recourse function $Q$, we can perturb the distribution of the underlying random variable in such a way that the resulting distribution has a pdf satisfying (6). This approach, which has been applied to pure integer recourse models in Klein Haneveld et al. (2006), Van der Vlerk (2004), will be extended to the current mixed-integer case below.

In principle, any suitable perturbation of the distribution can be used for this purpose, but—as in the pure integer case-we restrict to the following class.

Definition 1 For each $\alpha \in[0,1)$, the $\alpha$-approximation of a random variable $\varphi$ with cdf $F_{\varphi}$ is the continuous random variable $\varphi_{\alpha}$ with pdf $f_{\varphi_{\alpha}}$,

$$
f_{\varphi_{\alpha}}(t):=F_{\varphi}\left(\lfloor t\rfloor_{\alpha}+1\right)-F_{\varphi}\left(\lfloor t\rfloor_{\alpha}\right), \quad t \in \mathbb{R},
$$

where $\lfloor t\rfloor_{\alpha}:=\lfloor t-\alpha\rfloor+\alpha$ is the round down of $t$ with respect to $\alpha+\mathbb{Z}$.

Obviously, every pdf $f_{\varphi_{\alpha}}, \alpha \in[0,1)$, satisfies (6). With $F_{\varphi_{\alpha}}$ denoting the cdf of $\varphi_{\alpha}$, it is easy to see that $F_{\varphi_{\alpha}}(\alpha+k)=F_{\varphi}(\alpha+k), k \in \mathbb{Z}$. Further properties of $\alpha$-approximations are discussed in Klein Haneveld et al. (2006).

According to Corollary 1 , we can choose to either replace $\omega$ or $\delta$ by an $\alpha$-approximation. The resulting functions, defined for each $\alpha \in[0,1)$,

$$
Q_{\alpha}(x):=\mathbb{E}_{\delta_{\alpha}}\left[\left\lceil\delta_{\alpha}-x\right\rceil^{+}\right], \quad x \in \mathbb{R},
$$

and

$$
\begin{aligned}
Q^{\alpha}(x) & :=\mathbb{E}_{v}\left[\mathbb{E}_{\omega_{\alpha}}\left[\left\lceil\omega_{\alpha}-(x+v)\right\rceil^{+}\right]\right] \\
& =\mathbb{E}_{v}\left[G_{\alpha}(x+v)\right], \quad x \in \mathbb{R},
\end{aligned}
$$

are called $\alpha$-approximations of the recourse function $Q$; similarly, the function $G_{\alpha}$ is an $\alpha$ approximation of $G$. By construction, $Q_{\alpha}$ and $Q^{\alpha}$ are convex approximations of the mixedinteger recourse function $Q$. See Fig. 3.

As shown in Klein Haneveld et al. (2006), each $\alpha$-approximation $G_{\alpha}, \alpha \in[0,1)$, is piecewise linear and coincides with $G$ on the set $\{\alpha+\mathbb{Z}\}$. Hence, the same is true for the $\alpha$ approximations $Q_{\alpha}$, but not for $Q^{\alpha}$. Properties of the latter $\alpha$-approximations depend on the distribution of $v$, i.e., on the value function $v$.

Next we state bounds, uniform in $\alpha \in[0,1)$, for the respective approximation errors of $Q_{\alpha}$ and $Q^{\alpha}$, for the case that the random variables involved are continuously distributed. If $\delta=\omega-v$ is not continuously distributed, e.g., if $\omega$ is a discrete random variable and the value function $v$ is discontinuous, then we can only prove the trivial upper bound 1 for both approximation errors.

For $t \in \mathbb{R}$, let $\lceil t\rceil_{\alpha}:=\lceil t-\alpha\rceil+\alpha$ denote the round up of $t$ with respect to $\alpha+\mathbb{Z}$. 

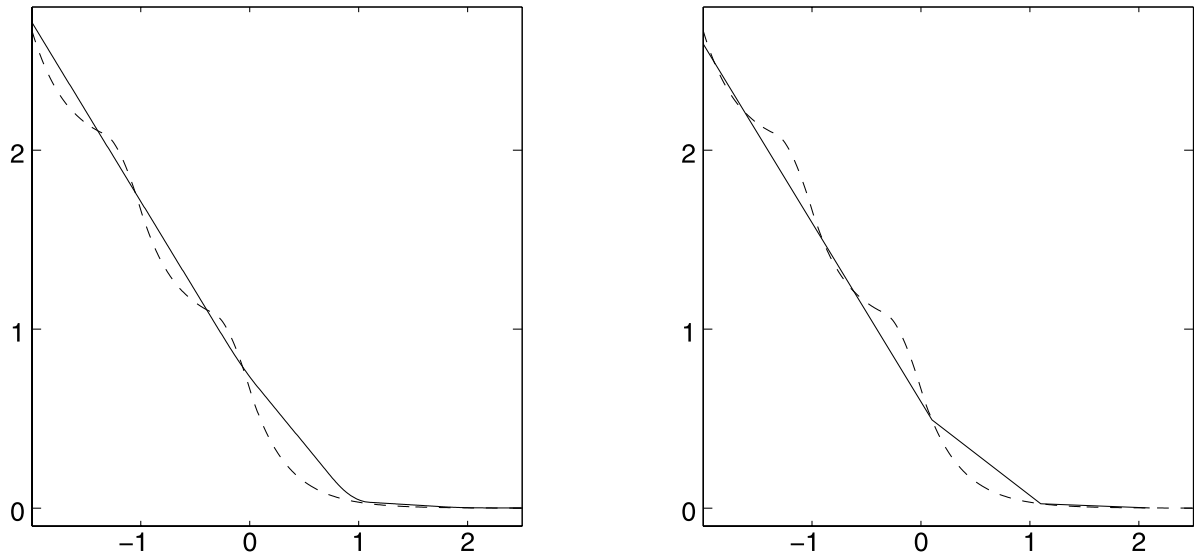

Fig. 3 The MISR function $Q$ (dashed) and the $\alpha$-approximations $Q^{\alpha}$ (left) and $Q_{\alpha}$ (right), for $q=3, \omega$ exponentially distributed with parameter 3 , and $\alpha=0.1$

Theorem 1 If $\delta=\omega-v$ has a pdf $f_{\delta}$ which is of bounded variation, then, for all $\alpha \in[0,1)$,

$$
\left|Q(x)-Q_{\alpha}(x)\right| \leq \min \left\{x-\lfloor x\rfloor_{\alpha},\lceil x\rceil_{\alpha}-x\right\} \frac{|\Delta| f_{\delta}}{2} \leq \frac{|\Delta| f_{\delta}}{4}, \quad x \in \mathbb{R},
$$

where $|\Delta| f_{\delta}$ denotes the total variation of $f_{\delta}$.

If $\omega$ has a pdf $f_{\omega}$ which is of bounded variation, then, for all $\alpha \in[0,1)$,

$$
\left|Q(x)-Q^{\alpha}(x)\right| \leq \min \left\{x-\lfloor x\rfloor_{\alpha},\lceil x\rceil_{\alpha}-x\right\} \frac{|\Delta| f_{\omega}}{2} \leq \frac{|\Delta| f_{\omega}}{4}, \quad x \in \mathbb{R} .
$$

Moreover, $|\Delta| f_{\delta} \leq|\Delta| f_{\omega}$. Thus, measured by the bounds above, the approximation of $Q$ by $Q_{\alpha}$ is at least as good as that by $Q^{\alpha}$.

Proof The bounds on the approximation errors follow immediately from the corresponding result for the function $G_{\alpha}$, see Klein Haneveld et al. (2006).

Referring to the definition of total variation, let $U:=\left\{u_{0}, u_{1}, \ldots, u_{N}\right\} \subset \mathbb{R}$ be such that

$$
|\Delta| f_{\delta}-\varepsilon<\sum_{i=1}^{N}\left|f_{\delta}\left(u_{i}\right)-f_{\delta}\left(u_{i-1}\right)\right|
$$

for every $\varepsilon>0$. Then

$$
\begin{aligned}
|\Delta| f_{\delta}-\varepsilon & <\sum_{i=1}^{N}\left|\int_{0}^{1}\left(f_{\omega}\left(u_{i}+s\right)-f_{\omega}\left(u_{i-1}+s\right)\right) d F_{v}(s)\right| \\
& \leq \int_{0}^{1} \sum_{i=1}^{N}\left|f_{\omega}\left(u_{i}+s\right)-f_{\omega}\left(u_{i-1}+s\right)\right| d F_{v}(s) \\
& \leq|\Delta| f_{\omega} .
\end{aligned}
$$


Remark 2 In most cases $Q_{\alpha}$ is a strictly better approximation of $Q$ compared to $Q^{\alpha}$, since the inequality (8) is strict unless the sets $\{U+s\}$ yield $|\Delta| f_{\omega}$ (in the sense of (7)) for $F_{v^{-}}$ almost all $s$.

Remark 3 Actually, the bounds derived in Theorem 1 apply to $\alpha$-approximations of the expectation of the scaled value function $v(p s) / v(p), s \in \mathbb{R}$, where $p$ is the period of the semi-periodic function $v$ restricted to $\mathbb{R}_{+}$. It follows by straightforward calculation that for the general case, the error bounds of Theorem 1 are multiplied by a factor $p v(p)$.

For many distributions, the total variation of the pdf decreases as the variance of the distribution increases. For example, the total variation of the normal distribution is proportional to the inverse of its standard deviation. Thus, we would expect that the $\alpha$-approximations $Q_{\alpha}$ and $Q^{\alpha}$ become better as the variance in the respective underlying distributions become larger.

\subsection{Continuous simple recourse representation}

It follows from the assumed semi-periodicity on $\mathbb{R}_{+}$of $v$, that every convex approximation $Q_{\alpha}(x)$ or $Q^{\alpha}(x), \alpha \in[0,1)$, has an asymptote (with slope -1 ) for $x \longrightarrow-\infty$. Moreover, both functions decrease to 0 as $x \longrightarrow-\infty$, so that each of them is Lipschitz continuous on $\mathbb{R}$. In Klein Haneveld et al. (1995) we showed that every such function can be represented as a continuous simple recourse function

$$
q^{+} \mathbb{E}_{\psi}\left[(\psi-x)^{+}\right]+q^{-} \mathbb{E}_{\psi}\left[(\psi-x)^{-}\right], \quad x \in \mathbb{R},
$$

with known distribution of the random variable $\psi$. Below we apply this result to the $\alpha$ approximations $Q_{\alpha}$ and $Q^{\alpha}$.

Corollary 2 For $\alpha \in[0,1)$, consider the $\alpha$-approximation

$$
Q_{\alpha}(x):=\mathbb{E}_{\delta_{\alpha}}\left[\left\lceil\delta_{\alpha}-x\right\rceil^{+}\right], \quad x \in \mathbb{R},
$$

where $\delta_{\alpha}$ is the $\alpha$-approximation of the random variable $\delta:=\omega-v, \omega$ has $c d f F_{\omega}$, and $v$ has $c d f F_{v}$.

$$
Q_{\alpha}(x)=\mathbb{E}_{\psi_{\alpha}}\left[\left(\psi_{\alpha}-x\right)^{+}\right], \quad x \in \mathbb{R},
$$

where the random variable $\psi_{\alpha}$ has cdf $\Psi_{\alpha}$,

$$
\Psi_{\alpha}(t)=\int_{0}^{1} F_{\omega}\left(\lfloor t\rfloor_{\alpha}+s\right) d F_{v}(s), \quad t \in \mathbb{R} .
$$

Since $\Psi_{\alpha}$ is constant on every interval $[\alpha+k, \alpha+k+1), k \in \mathbb{Z}$, the random variable $\psi_{\alpha}$ is discrete with support in $\{\alpha+\mathbb{Z}\}$.

Corollary 3 For $\alpha \in[0,1)$, consider the $\alpha$-approximation

$$
Q^{\alpha}(x):=\mathbb{E}_{v}\left[\mathbb{E}_{\omega_{\alpha}}\left[\left\lceil\omega_{\alpha}-(x+v)\right\rceil^{+}\right]\right], \quad x \in \mathbb{R},
$$

where $\omega_{\alpha}$ is the $\alpha$-approximation of the random variable $\omega$ with $c d f F_{\omega}$, and $v$ has cdf $F_{v}$.

$$
Q^{\alpha}(x)=\mathbb{E}_{\varphi_{\alpha}}\left[\left(\varphi_{\alpha}-x\right)^{+}\right], \quad x \in \mathbb{R},
$$


where the random variable $\varphi_{\alpha}$ has $c d f \Phi_{\alpha}$,

$$
\Phi_{\alpha}(t)=\int_{0}^{1} F_{\omega}\left(\lfloor t+s\rfloor_{\alpha}\right) d F_{v}(s), \quad t \in \mathbb{R} .
$$

\section{Solving mixed-integer recourse models}

Returning to the recourse function $\mathcal{Q}(x)=\mathbb{E}_{\omega}[v(\omega-T x)], x \in \mathbb{R}^{n}$, we conclude that convex approximations can be obtained by $\alpha$-approximations. Moreover, each such function $\mathcal{Q}_{\alpha}(x)$ or $\mathcal{Q}^{\alpha}(x), \alpha \in[0,1)$, can be represented as a continuous simple recourse function with random right-hand side parameter, whose distribution is known. In particular, for each function $\mathcal{Q}_{\alpha}$ the corresponding distribution is discrete.

Hence, to approximately solve the mixed-integer recourse problem

$$
\min _{x \in X} c x+\mathcal{Q}(x)
$$

we can solve instead the continuous simple recourse problem

$$
\min _{x \in X} c x+\mathcal{Q}_{\alpha}(x)
$$

or

$$
\min _{x \in X} c x+\mathcal{Q}^{\alpha}(x)
$$

for one or more values of the parameter $\alpha$. If all first-stage variables are continuous, then this can be done by existing algorithms (see e.g. Mayer 1998), which are very efficient. If not, then one could apply a branch and bound scheme, and use these algorithms to solve subproblems.

It is easy to see that the bounds presented in Theorem 1 also apply to the respective approximation errors in the optimal values of (10) and (11). Depending on the application, this guarantee on the approximation error may be satisfactory or not. In the latter case, we may use that each $\alpha$-approximation yields a convex lower bound for $\mathcal{Q}$ (polyhedral in case of $\mathcal{Q}_{\alpha}$ ) on substraction of the corresponding approximation error given in Theorem 1. Such convex lower bounding approximations can be exploited in branch-and-bound methods to solve the problem (9) exactly. Alternatively, one could solve approximating problems (10) or (11) for a number of parameter values $\alpha$, yielding respective optimal solutions $x_{\alpha}$, and compare them by calculating the true objective values $c x_{\alpha}+\mathcal{Q}\left(x_{\alpha}\right)$.

As we will show next, the evaluation of $\mathcal{Q}\left(x_{\alpha}\right)$ may not be necessary if $x_{\alpha}$ is an optimal solution of (10). For each $\alpha \in[0,1)$, the random variable $\psi_{\alpha}$ underlying the continuous simple recourse function $\mathcal{Q}_{\alpha}$ is discrete with support in $\alpha+\mathbb{Z}$ by Corollary 2. As is well known, the function $\mathcal{Q}_{\alpha}$ is therefore polyhedral, and it is non-linear at $x$ if and only if $T x$ belongs to the support of $\psi_{\alpha}$. Hence, if $x_{\alpha}$ is a free optimal solution of (10), then $T x_{\alpha} \in$ $\alpha+\mathbb{Z}$, so that $\mathcal{Q}_{\alpha}\left(x_{\alpha}\right)=\mathcal{Q}\left(x_{\alpha}\right)$ as discussed in Sect. 3.2.

For this reason, and moreover since

(i) solving (10) is in general easier than solving (11), which according to Corollary 3 involves a random variable with arbitrary distribution type;

(ii) by Theorem 1 and Remark 2, the approximation obtained by (10) is at least as good as that of (11),

we conclude that the convex approximations $\mathcal{Q}_{\alpha}$ are preferable over $\mathcal{Q}^{\alpha}$ for the purpose of (approximately) solving the mixed-integer recourse model (9). 


\section{Summary and concluding remarks}

For a restricted class of mixed-integer recourse models, we showed that the second-stage value function $v$ is equivalent to an expected integer shortage function $H$, where the expectation is taken with respect to a distribution which reflects the particular value function. Thus, the mixed-integer recourse function $\mathcal{Q}(x):=\mathbb{E}_{\omega}[v(\omega-T x)], x \in \mathbb{R}^{n}$, can be seen as the expectation of $H(\omega-T x)$, allowing to derive its properties from those of $H$, which is well-studied in the context of simple integer recourse models.

In particular, we showed that convex approximations of $\mathcal{Q}$ can be obtained by suitable perturbations of the distributions involved. This approach, first developed for simple integer recourse models (Klein Haneveld et al. 2006) and later extended to general pure integer recourse models (Van der Vlerk 2004), is thus shown to be applicable to some mixed-integer recourse models too.

Next, we showed that the convex approximations obtained can be represented as recourse functions of continuous simple recourse models. Thus, instead of solving such a mixedinteger recourse model directly, we can

(i) modify the recourse structure (in particular, get rid of integrality constraints in the second-stage problem), and

(ii) perturb the underlying distribution,

to obtain a continuous simple recourse model which is easy to solve, and provides an approximate solution of the original model. The results presented in this paper extend this modification of recourse data approach (Van der Vlerk 2003), which was previously applied to pure integer (and continuous multiple simple) recourse models, to a class of mixed-integer recourse models.

In future research we hope to further extend this approach to more general mixed-integer recourse models.

Acknowledgement I am grateful to Wim Klein Haneveld for many beneficial discussions.

Open Access This article is distributed under the terms of the Creative Commons Attribution Noncommercial License which permits any noncommercial use, distribution, and reproduction in any medium, provided the original author(s) and source are credited.

\section{References}

Alonso-Ayuso, A., Escudero, L. F., \& Ortuño, M. T. (2003). BFC, a branch-and-fix coordination algorithmic framework for solving some types of stochastic pure and mixed 0-1 programs. European Journal of Operational Research, 151(3), 503-519.

Birge, J. R., \& Louveaux, F. V. (1997). Introduction to stochastic programming. New York: Springer.

Carøe, C. C., \& Tind, J. (1997). A cutting-plane approach to mixed 0-1 stochastic integer programs. European Journal of Operational Research, 101(2), 306-316.

Dyer, M., \& Stougie, L. (2006). Computational complexity of stochastic programming problems. Mathematical Programming, 106(3, Ser. A), 423-432.

Guan, Y., Ahmed, S., \& Nemhauser, G. L. (2005). Sequential pairing of mixed integer inequalities. In Lecture notes in comput. sci.: Vol. 3509. Integer programming and combinatorial optimization (pp. 23-34). Berlin: Springer.

Kall, P., \& Wallace, S. W. (1994). Stochastic programming. New York: Wiley. Also available as PDF file e.g. via http://stoprog.org.

Klein Haneveld, W. K., \& van der Vlerk, M. H. (1999). Stochastic integer programming: general models and algorithms. Annals of Operation Research, 85, 39-57. 
Klein Haneveld, W. K., Stougie, L., \& van der Vlerk, M. H. (1995). On the convex hull of the simple integer recourse objective function. Annals of Operation Research, 56, 209-224.

Klein Haneveld, W. K., Stougie, L., \& van der Vlerk, M. H. (1996). An algorithm for the construction of convex hulls in simple integer recourse programming. Annals of Operation Research, 64, 67-81.

Klein Haneveld, W. K., Stougie, L., \& van der Vlerk, M. H. (2006). Simple integer recourse models: convexity and convex approximations. Mathematical Programming, 108(2-3), 435-473.

Kong, N., Schaefer, A. J., \& Hunsaker, B. (2006). Two-stage integer programs with stochastic right-hand sides: a superadditive dual approach. Mathematical Programming, 108(2-3), 275-296.

Laporte, G., \& Louveaux, F. V. (1993). The integer L-shaped method for stochastic integer programs with complete recourse. Operations Research Letters, 13, 133-142.

Louveaux, F. V., \& Schultz, R. (2003). Stochastic integer programming. In A. Ruszczynski \& A. Shapiro (Eds.), Handbooks in operations research and management science: Vol. 10. Stochastic programming (Chap. 4, pp. 213-266). Amsterdam: Elsevier.

Louveaux, F. V., \& van der Vlerk, M. H. (1993). Stochastic programming with simple integer recourse. Mathematical Programming, 61, 301-325.

Mayer, J. (1998). Optimization theory and applications: Vol. 1. Stochastic linear programming algorithms: a comparison based on a model management system. New York: Gordon and Breach.

Norkin, V. I., Ermoliev, Yu. M., \& Ruszczyński, A. (1998). On optimal allocation of indivisibles under uncertainty. Operational Research, 46(3), 381-395.

Prékopa, A. (1995). Stochastic programming. Dordrecht: Kluwer Academic.

Ruszczynski, A., \& Shapiro, A. (2003). Handbooks in operations research and management science: Vol. 10. Stochastic programming. Amsterdam: Elsevier.

Schultz, R. (1993). Continuity properties of expectation functions in stochastic integer programming. Mathematics of Operations Research, 18, 578-589.

Schultz, R. (1995). On structure and stability in stochastic programs with random technology matrix and complete integer recourse. Mathematical Programming, 70, 73-89.

Schultz, R., \& Tiedemann, S. (2003). Risk aversion via excess probabilities in stochastic programs with mixed-integer recourse. SIAM Journal of Optimization (electronic), 14(1), 115-138.

Schultz, R., \& Tiedemann, S. (2006). Conditional value-at-risk in stochastic programs with mixed-integer recourse. Mathematical Programming, 105(2-3), 365-386.

Sen, S., \& Higle, J. L. (2005). The $\mathrm{C}^{3}$ theorem and a $\mathrm{D}^{2}$ algorithm for large scale stochastic mixed-integer programming: set convexification. Mathematical Programming, 104(1), 1-20.

Sen, S., \& Sherali, H. D. (2006). Decomposition with branch-and-cut approaches for two-stage stochastic mixed-integer programming. Mathematical Programming, 106(2), $203-223$.

Sherali, H. D., \& Zhu, X. (2006). On solving discrete two-stage stochastic programs having mixed-integer first- and second-stage variables. Mathematical Programming, 108(2-3), 597-616.

Silva, F., \& Wood, R. K. (2006). Solving a class of stochastic mixed-integer programs with branch and price. Mathematical Programming, 108(2-3), 395-418.

Stougie, L., \& van der Vlerk, M. H. (1997). Stochastic integer programming. In M. Dell' Amico \& F. Maffioli (Eds.), Annotated bibliographies in combinatorial optimization (Chap. 9, pp. 127-141). New York: Wiley.

Stougie, L., \& van der Vlerk, M. H. (2003). Approximation in stochastic integer programming. Research Report 2003-12, Stochastic Programming E-Print Series, http://www.speps.org.

Swamy, C., \& Shmoys, D. B. (2005). Sampling-based approximation algorithms for multi-stage stochastic optimization. In 46th annual IEEE symposium on foundations of computer science (FOCS'05) (pp. 357366)

van der Vlerk, M. H. (1995). Stochastic programming with integer recourse. Ph.D. thesis, University of Groningen, The Netherlands.

van der Vlerk, M. H. (2003). Simplification of recourse models by modification of recourse data. In K. Marti \& Y. Ermoliev (Eds.), Lecture notes in economics and mathematical systems: Vol. 532. Dynamic stochastic optimization (pp. 321-336). Berlin: Springer.

van der Vlerk, M. H. (2004). Convex approximations for complete integer recourse models. Mathematical Programming, 99(2), 297-310. 